\title{
Crisis, cambio en la UE y estrategias sindicales: el impacto de la condicionalidad en el repertorio estratégico de los sindicatos españoles durante la crisis de la eurozona
}

Crisis, EU transformation and union strategies: The impact of conditionality on the strategic repertoire of Spanish trade unions during the Eurozone crisis

\author{
ANGIE GAGO \\ Universidad de Milán

\section{Cómo citar/Citation} \\ Gago, A. (2016). Crisis, cambio en la UE y estrategias sindicales: el impacto de la condicionalidad en el repertorio estratégi- \\ co de los sindicatos españoles durante la crisis de la eurozona. Revista Española de Ciencia Política, 42, 45-68. Doi: hittp:// \\ dx.doi.org/10.21308/recp.42.02
}

\section{Resumen}

Durante la crisis de la eurozona, los gobiernos del PSOE y del PP implementaron una serie de reformas del Estado de Bienestar y del mercado laboral a cambio de ayuda financiera por parte de la Unión Europea, lo que se conoce como condicionalidad. Los sindicatos, CC. OO. y UGT, respondieron ante tales reformas como representantes de los colectivos más afectados por las mismas. Existen dos argumentos distintos que explican las estrategias de los sindicatos durante la crisis. Por una parte, se argumenta que los sindicatos utilizaron la acción política tradicional para influir en los procesos de toma de decisiones. Por otra parte, los sindicatos han puesto en marcha nuevas acciones bien de carácter transnacional o relacionadas con actividades propias de los movimientos sociales. El artículo explica por qué la crisis dio lugar a esta nueva combinación de repertorios sindicales que apelan a sectores y niveles de acción distintos. El argumento principal es que la condicionalidad de la UE dio lugar a la aparición de un nuevo régimen de decisión política que denominamos aquí «intergubernamentalismo neoliberal» por los cambios institucionales e ideológicos que trajo consigo. A su vez este nuevo régimen impactó en la estructura de oportunidad política, provocando una reorientación estratégica de los sindicatos.

Palabras clave: Unión Europea, crisis económica, condicionalidad, neoliberalismo, intergubernamentalismo, corporatismo, sindicatos, estrategias 


\begin{abstract}
During the eurozone crisis, the PSOE's and PP's governments implemented a series of reforms of the Welfare State and the labour market in exchange for financial help from the EU; what is known as conditionality. The trade unions, CC. OO. and UGT, responded to these reforms as the representatives of the sectors most affected by them. There are two narratives to explain trade unions' strategies during the crisis. On the one hand, it is argued that trade unions used traditional political action to influence political decision making processes. On the other hand, trade unions have developed new actions either of a transnational character or related to typical social movements' activities. The article explains why the crisis led to this new combination of trade union repertories calling on different sectors and levels of action. The main argument is that the EU conditionality led to the emergence of a new political regime that we have dubbed "neoliberal intergovernmentalism" due to the institutional and ideological changes that it brought about. In turn, this new regime impacted upon the political opportunity structure, triggering a strategic reorientation of trade unions.
\end{abstract}

Key words: European Union, crisis, conditionality, neoliberalism, intergovernmentalism, corporatism, trade unions, strategies

\title{
INTRODUCCIÓN
}

Durante la crisis de la eurozona (2010-2013) los Gobiernos del Partido Socialista Obrero Español (PSOE) y del Partido Popular (PP) implementaron una serie de reformas del Estado de bienestar y del mercado laboral. Estas provocaron la respuesta de los sindicatos mayoritarios españoles, Comisiones Obreras (CC. OO.) y la Unión General de Trabajadores (UGT), como representantes de los sectores de la sociedad más afectados por las mismas. Los estudios sobre las respuestas sindicales frente a las políticas de austeridad presentan dos argumentos diferentes. Una primera perspectiva señala que las organizaciones sindicales se vieron afectadas por su expulsión de los procesos de formulación de política pública (González Begega y Luque Balbona, 2014, 2015; Molina y Miguélez, 2013). Sabemos que los sindicatos respondieron ante este hecho utilizando el repertorio tradicional de acción política basado en el diálogo social (en las pocas ocasiones en las que los Gobiernos optaron por la concertación) y las huelgas generales (González Begega y Luque Balbona, 2015; Campos Lima y Martin Artiles, 2011; Hyman, 2010). CC. OO. y UGT firmaron el Acuerdo Social y Económico para el Crecimiento, el Empleo y la Garantía de las Pensiones en febrero de 2011 con el Gobierno del PSOE, contra el que también convocaron una huelga general en septiembre de 2010 contra la reforma laboral. Asimismo, los sindicatos organizaron otras dos huelgas generales contra el Gobierno del PP en marzo y noviembre de 2012.

Por el contrario, un segundo enfoque apunta a que la crisis de la eurozona forzó a los sindicatos a realizar una reorientación estratégica y a explorar nuevos repertorios de acción. Por una parte, pusieron en marcha acciones transnacionales, siendo el punto más álgido la huelga general europea del 14 de noviembre de 2012, en cuyo desarrollo 
tuvieron un papel crucial los sindicatos españoles (Dribbusch, 2015; Helle, 2015). Por otra parte, CC. OO. y UGT «redescubrieron un modelo de protesta que trasciende la representación de los intereses de los trabajadores y conecta con la sociedad civil» (González Begega y Luque Balbona, 2014: 97). Así, los sindicatos lanzaron una iniciativa legislativa popular (ILP) en junio de 2010 para proponer un cambio de modelo de relaciones laborales, participaron en las mareas por la defensa de los servicios públicos en 2011, crearon la cumbre social junto con otras asociaciones de la sociedad civil, y lanzaron una campaña para la realización de un referéndum contra las medidas de austeridad del PP en el verano de 2012.

Además, todos estos estudios apuntan a que la imposición por parte de la Unión Europea de reformas del Estado de bienestar y del mercado de trabajo a cambio de ayuda financiera, lo que se conoce como "condicionalidad», es una variable fundamental para entender las respuestas de los sindicatos durante la crisis. En los países que solicitaron el rescate financiero como Portugal o Grecia, la condicionalidad se desarrolló de manera formal y los memorándum de entendimiento contenían explícitamente la demanda de realizar recortes en las políticas sociales y reformas encaminadas a desregularizar el mercado de trabajo. Por el contrario, en España, durante el Gobierno del PSOE (2010-2011) la condicionalidad se desarrolló de manera informal a través de los "cuartos traseros de la diplomacia» (De la Porte y Natali, 2014: 735), como las llamadas de teléfono entre el expresidente José Luis Rodríguez Zapatero y la canciller alemana, Angela Merkel, o la carta enviada por el presidente del Banco Central Europeo (BCE), Jean-Claude Trichet, al expresidente socialista en agosto de 2011. En una segunda etapa, durante el Gobierno del PP (2012-2013), a los "cuartos traseros» hay que añadirle la condicionalidad adjunta al rescate bancario de junio de 2012. Aunque el memorando solo contenía medidas para reformar el mercado financiero, también incluía un proceso de monitoreo paralelo y de «estrecha supervisión» de las reformas estructurales incluidas en el Procedimiento de Déficit Excesivo y en el Semestre Europeo ${ }^{1}$.

Este artículo tiene como objetivo explicar por qué los sindicatos españoles combinaron el repertorio clásico de acción política con acciones transnacionales y otras actividades orientadas a las demandas de derechos civiles frente a la aparición de la condicionalidad de la Unión Europea. Para responder a esta pregunta el artículo utiliza el método de rastreo de procesos causales. Este método, concretamente su variante denominada "construcción de teorías», es útil cuando tenemos una variable independiente (condicionalidad de la Unión Europea) y una variable dependiente (la reorientación estratégica de los sindicatos), pero desconocemos los mecanismos y los procesos existentes entre las dos (Beach y Pedersen, 2013:16).

1. Memorando de Entendimiento sobre Condiciones de Política Sectorial Financiera, hecho en Bruselas y Madrid el 23 de julio de 2012, y Acuerdo Marco de Asistencia Financiera, hecho en Madrid y Luxemburgo el 24 de julio de 2012. Boletín Oficial del Estado, núm. 296, lunes 10 de diciembre de 2012, sec. I. pág. 84550. 
A través de un seguimiento de los procesos de adopción de decisiones en las instituciones europeas y de los procesos de formulación de políticas públicas, el artículo construye un mecanismo causal que conecta ambas variables. La construcción de dicho mecanismo se sustenta en la inferencia causal de diferentes tipos de observaciones empíricas como la literatura secundaria, fuentes primarias (informes de los sindicatos, actas de reuniones, relatos periodísticos, libros de memorias, etc.) y las entrevistas personales semiestructuradas que la autora del artículo realizó entre noviembre de 2015 y marzo de 2016 a líderes sindicales y miembros del Ministerio de Trabajo. El argumento principal del artículo es que la condicionalidad de la UE dio lugar a la aparición de un nuevo régimen político de adopción de decisiones que denominamos aquí «intergubernamentalismo neoliberal» (INL) por el tipo de cambios institucionales e ideológicos que trajo consigo. Este nuevo régimen político provocó también un nuevo modo de formulación de políticas públicas. Todos estos cambios modificaron a su vez la estructura de oportunidad política de la que disponen los sindicatos para ejercer influencia en los procesos políticos. La acción política de los sindicatos perdió su significado y estos se vieron forzados a realizar una reorientación estratégica.

La aportación de este artículo es relevante tanto por motivos teóricos como políticos. Primero, porque desarrolla instrumentos de análisis que nos permiten entender el comportamiento de los sindicatos en un escenario político y económico nuevo. El marco analítico propuesto se inspira en la literatura de movimientos sociales ya que conecta la existencia de regímenes políticos con una estructura de oportunidad política y repertorios estratégicos específicos (Tilly y Tarrow, 2015). Segundo, porque el INL suscita debates en torno su legitimidad democrática. El nuevo modo de decisiones desarrollado durante la crisis ignora las instituciones políticas domésticas y los mecanismos para agregar las demandas de los grupos de intereses (Armingeon y Baccaro, 2012). Esto último tiene consecuencias políticas importantes para los sindicatos, ya que en un nuevo escenario político y económico neoliberal, en el que las decisiones se toman en espacios intergubernamentales y fuera de los cauces de la democracia nacional representativa, los sindicatos parecen perder parte de su rol tradicional.

El artículo está dividido en tres secciones. La primera sección realiza un breve resumen de las explicaciones existentes en la literatura académica sobre las respuestas de los sindicatos españoles durante la crisis. La segunda propone un marco analítico para explicar cómo y bajo qué condiciones la condicionalidad de la UE impactó en las estrategias de los sindicatos. En una primera parte se describen los conceptos centrales del marco analítico para después, en una segunda parte, aplicar dichos conceptos a lo acontecido durante la crisis de la eurozona. Se explica aquí la aparición de un nuevo régimen político, el INL neoliberal, y su impacto en la estructura de oportunidad política. La tercera sección presenta un estudio de caso de los procesos de formulación de política pública desde 2010 a 2013 respecto a las reformas de las pensiones y del mercado laboral. El objetivo de esta sección es ilustrar el funcionamiento del mecanismo causal en el caso español. La cuarta sección presenta algunas conclusiones. 


\section{LAS ESTRATEGIAS DE LOS SINDICATOS ESPAÑOLES DURANTE LA CRISIS DE LA EUROZONA}

Las respuestas de los sindicatos españoles durante la crisis han sido ampliamente analizadas por la literatura académica. Como hemos apuntado anteriormente, los estudios presentan dos argumentos diferentes. Por una parte, los sindicatos habrían recurrido a la acción política para responder a las reformas del Estado de bienestar y del mercado laboral implementadas tanto por el Gobierno del PSOE como del PP. En relación con esto, la mayoría de los estudios coinciden en que durante la crisis la mayoría de las reformas fueron implementadas de forma unilateral por parte de los Gobiernos, provocando esto la expulsión de los sindicatos de los procesos de formación de políticas públicas (González Begega y Luque Balbona, 2014, 2015; Molina y Miguélez, 2013).

En España no se ha llegado nunca a consolidar un sistema de intermediación de intereses de estilo corporativista (Schmitter, 1974). Este tipo de sistemas es típico en las economías coordinadas de mercado de los países nórdicos y de algunos países de Europa continental. En estos países los derechos laborales y políticos de los sindicatos relacionados con la codeterminación de las políticas públicas y del mercado laboral están institucionalizados (Hall y Soskice, 2001). Por otra parte, España tampoco se considera una economía liberal de mercado en el que los sindicatos no tienen ningún papel político. Esto se debe a que, a pesar de la inexistencia de instituciones formales, se han desarrollado procesos similares al corporatismo, lo que se conoce como concertación (Molina y Rhodes, 2002; Baccaro, 2003). En este tipo de "corporatismo débil» (Oliet 2004; Molina 2005), también conocido como "economía de mercado mixto", los Gobiernos han incluido tradicionalmente a los sindicatos en procesos de concertación para obtener su consenso ante medidas impopulares y evitar así el poder de veto que estos pueden ejercer a través de la movilización (Ebbinghaus, 2011). Esto último ha facilitado la participación de los sindicatos españoles como actores políticos.

Sin embargo, durante la crisis solo los Gobiernos de los países nórdicos y de otros países de Europa continental se han enfrentado a los efectos de la crisis recurriendo a la colaboración con los sindicatos, mientras que la erosión del corporatismo, también denominada "post-corporatismo", ha sido un fenómeno generalizado en los países del Sur de Europa e Irlanda, es decir, en los países que durante la crisis de deuda necesitaron ayuda financiera por parte de la UE (Urban, 2012).

Esto ha llevado a pensar a que la imposición de las medidas de austeridad por parte de la UE a los países de la periferia es una variable crucial para explicar el descenso del corporatismo durante crisis. De hecho, el debate sobre si la UE facilita u obstaculiza el corporatismo ya estaba abierto antes de la crisis aunque con conclusiones divergentes (Bieler y Morton, 2001; Afonso, 2013; Hyman 2001, 2005; Erne, 2008). Por una parte, algunos autores argumentan que la integración europea ha reducido las competencias de los Gobiernos nacionales en torno a cuestiones económicas (Scharpf, 2002). La pérdida de soberanía ha dado lugar a un descenso del corporatismo porque los Gobiernos no tienen recursos para ofrecer concesiones a los sindicatos en la negociación (Bieler y Morton, 2001; Afonso, 2013). 
Por el contrario, a mediados de los noventa hubo un aumento de los pactos sociales entre Gobiernos y sindicatos en el Sur de Europa con el objetivo de cumplir los requisitos fiscales para entrar en la Unión Económica y Monetaria (UEM) (Avdagic et. al., 2011). Para explicar este resultado diferente, aumento o descenso del corporatismo ante un mismo estímulo, algunos autores señalan que el corporatismo no es tanto una respuesta funcional ante un problema económico sino una estrategia política que está determinada por los intereses y la interacción de los actores políticos y las dinámicas institucionales (Afonso, 2013; Avdagic et al., 2011; Molina, 2005; Natali y Pochet, 2009; Hamann, 2012). En este sentido, la concertación depende más de si los Gobiernos son mayoritarios o minoritarios o de sus cálculos electorales (Afonso, 2013; Hamann y Kelly, 2007). En relación con esto último, la literatura señala que los gobiernos utilizan la concertación como estrategia de "evitación de la culpa» (blame avoidance) para evitar ser penalizados en las elecciones, transfiriendo la responsabilidad de la implementación de reformas impopulares a la esfera corporativista (Weaver, 1986).

Sin embargo, la crisis de la eurozona no ha dado lugar a un aumento de los pactos sociales como ocurrió a mediados de los noventa. Esto tuvo como consecuencia un aumento de la conflictividad por parte de los sindicatos del Sur del Europa desde 2010 a 2013. El aumento de huelgas generales coincidió con la realización de numerosas manifestaciones (Campos Lima y Martín Artiles, 2014). En general, la literatura indica que los sindicatos utilizan las huelgas generales para apelar a los Gobiernos a la negociación o para presionarles cuando las negociaciones se han visto frustradas por falta de acuerdo (Kelly y Hamann, 2004; Afonso, 2013; Campos Lima y Martín Artiles, 2011). Sin embargo, otros estudios más recientes apuntan a que las huelgas generales han cambiado de significado durante la crisis de la eurozona. Estas fueron utilizadas no solo para abrir una confrontación directa con los Gobiernos nacionales sino también para protestar contra la austeridad impuesta por la UE, adoptando así un carácter transnacional (Helle, 2015).

Esto conecta con la segunda perspectiva planteada en la literatura académica, desde la cual se argumenta que los sindicatos han explorado repertorios de acción alternativos basados en acciones transnacionales y otras encaminadas a conectar con amplios sectores de la sociedad civil. Respecto a lo primero, algunos autores afirman que los sindicatos españoles tuvieron especial importancia en la organización de lo que se ha denominado la primera huelga general europea del 14 de noviembre de 2012 (Dribbusch, 2015; Helle, 2015). El papel de CC. OO. y UGT dentro de la Confederación Europea de Sindicatos (CES) fue crucial para la convocatoria de una huelga conjunta que también fue asumida por sindicatos en Portugal o Italia. Además, las huelgas generales han servido también a los sindicatos para politizar a amplios sectores de la sociedad más allá de las bases sindicales. En este hecho ha tenido una importancia especial la simbiosis creada entre sindicatos y movimientos sociales tras la aparición de movimientos como los indignados o el 15M (Cerrillo Vidal, 2013; Campos Lima y Martin Artiles, 2014).

Por último, todos estos estudios otorgan también un papel crucial a la crisis económica para entender la emergencia de estrategias alternativas. Por una parte, el hecho 
de que las reformas fuesen impuestas por la UE, hizo que sindicatos de diferentes países intentaran articular una respuesta conjunta. Por otra parte, el impacto de la crisis en los sistemas democráticos cuestionó el papel de los sindicatos en la gobernanza económica forzándolos a ampliar sus bases de apoyo a través de acciones abiertas a la sociedad civil (Barranco y Molina, 2014). Entonces, ¿cómo es posible que la crisis de la eurozona diera lugar a la puesta en marcha por parte de los sindicatos de repertorios estratégicos distintos que apelan a diferentes colectivos y niveles de acción?

\section{UNA PROPUESTA DE MARCO ANALÍTICO: LA ESTRUCTURA DE OPORTUNIDAD POLÍTICA}

Figura 1.

Marco ANALÍtico

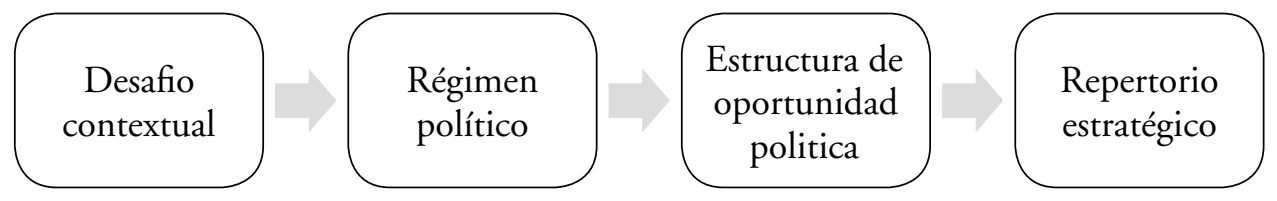

Fuente: elaboración propia.

El concepto de «estructura de oportunidad política» (EOP), desarrollado por la literatura de los movimientos sociales (Tilly, 1995), es fundamental para explicar los diferentes niveles y tipos de estrategias disponibles para los sindicatos en un contexto concreto (Princen y Kerremans, 2008: 1131). Este artículo propone un marco analítico que nos permite explicar cómo y bajo qué condiciones el desafío contextual de la crisis impactó en la reorientación estratégica de los sindicatos. Este marco establece una relación entre la existencia de regímenes políticos concretos con un tipo de estructura de oportunidad política específica. Tilly y Tarrow consideran que los regímenes son «las relaciones regulares entre los Gobiernos, los actores políticos establecidos y rivales, y son percibidos y cumplidos por actores políticos externos, incluyendo otros Gobiernos» (Tilly y Tarrow, 2015: 49).

Por su parte, el concepto de EOP se refiere al grado de apertura o cierre de los regímenes políticos a nuevas demandas (Tilly, 1995). Aunque originalmente fue utilizado para explicar la capacidad de los movimientos sociales para desarrollar acciones de protesta, más tarde también ha sido utilizado para analizar las estrategias de los sindicatos (Hyman y Gumbrell-McCormick, 2010; Streeck y Hassel, 2003). Kitschelt define la EOP como la «específica configuración de recursos, arreglos institucionales y antecedentes históricos para la movilización social, los cuales facilitan el desarrollo de los movimientos de protesta en algunas ocasiones y los constriñen en otras» (Kitschelt, 1986: 58). En este artículo consideramos que la EOP se puede entender como un subsistema del régimen político compuesto por los elementos del 
mismo que nos ayudan a explicar cómo los sistemas políticos facilitan o limitan las acciones de los sindicatos. Por último, la literatura de movimientos sociales apunta que cada EOP define un repertorio estratégico específico para la intervención de los sindicatos. Tilly define el concepto de repertorio como un «conjunto completo de acciones y herramientas de organización y protesta» (Tilly 2006). Aun así, la EOP no determina el comportamiento de los sindicatos ya que es creada al mismo tiempo por las acciones de los mismos (Princen y Kerremans, 2008).

La condicionalidad de la Unión Europea y la aparición de un nuevo régimen político: intergubernamentalismo neoliberal

Figura 2.

MECANISMO CAUSAL

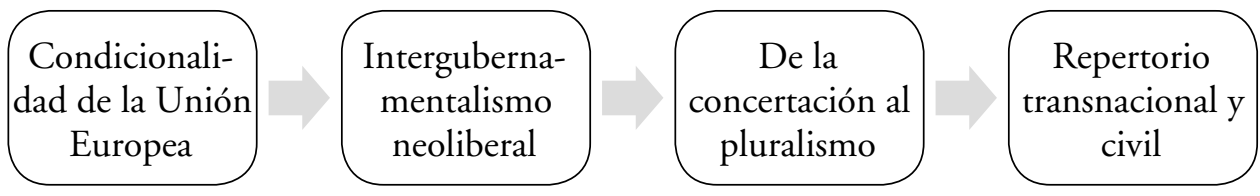

Fuente: elaboración propia.

Esta sección aplica el marco analítico presentado anteriormente a las condiciones y dinámicas políticas desarrolladas durante la crisis de la eurozona. En este sentido, argumentamos que el desafío contextual de la condicionalidad de la UE provocó la aparición de un nuevo régimen político que denominamos «intergubernamentalismo neoliberal» (INL). Este régimen político trajo consigo una serie de cambios institucionales e ideológicos, y un nuevo modo de formulación de políticas públicas. Una de las consecuencias principales de la condicionalidad fue el aumento del intergubernamentalismo (Degryse, 2012; Schimmelfennig, 2015a). La gestión de la crisis se llevó a cabo en instituciones y foros intergubernamentales como el Eurogrupo, el Consejo Europeo y el Consejo de Asuntos Económicos y Financieros (Ecofin), mientras que las instituciones supranacionales como la Comisión Europea tuvieron un papel residual (Degryse, 2012). Además tanto el Mecanismo Europeo de Estabilidad como el Pacto Fiscal Europeo fueron tratados intergubernamentales (Schimmelfennig, 2015a).

Esto revivió el debate entre dos de las teorías principales de la integración europea en torno al tipo de régimen político existente en la UE. Por una parte, la teoría supranacionalista argumenta que el proyecto europeo se expande gracias al efecto spillover de un área a otra (Haas, 1958). Por el contrario, el intergubernamentalismo sostiene que los intereses nacionales son fundamentales para entender los procesos de expansión y contracción de la UE. Este fue más tarde reformulado por Moravcsik como INL (Moravcsik, 1993). Este artículo no pretende crear una teoría de la integración europea alternativa a la de Moravcsik sino recuperar algunos elementos de la misma y 
adaptar otros al contexto creado por la condicionalidad para explicar por qué no podemos hablar más de un régimen intergubernamental liberal sino neoliberal.

Sostenemos aquí que la forma en la que se desarrolló la condicionalidad europea cuestiona algunos de los supuestos de la teoría de Moravcsik. Para explicar la aparición de la condicionalidad algunos autores subrayan la existencia de dos grupos diferenciados dentro del Consejo Europeo. Por una parte, el grupo de los países acreedores liderados por Alemania y, por otra, el grupo de los deudores formado principalmente por los países del Sur de Europa. Aunque ambos grupos compartían el objetivo final de mantener el euro, mantenían opiniones divergentes sobre cómo conseguir la credibilidad de los mercados financieros (Schimmelfennig, 2015a). Mientras que los países con dificultades pedían soluciones basadas en la solidaridad como la emisión de los eurobonos, el grupo con más poder, los acreedores, identificó la deuda pública y las «rigideces» del mercado laboral como las causas principales de la crisis. Como consecuencia, impusieron una estrategia de devaluación interna a los deudores (Armingeon y Baccaro, 2012).

Así nació la condicionalidad: a cambio de ayuda financiera para bajar la especulación, los países del Sur de Europa tenían que implementar políticas de austeridad y reformas estructurales basadas en los recortes de las políticas sociales y la desregulación del mercado de trabajo. Este proceso, que después ha sido institucionalizado a través de la creación del Mecanismo Europeo de Estabilidad Financiera, contradice las premisas del INL. Este argumenta que la coordinación política entre países tiene que ser voluntaria, sin amenazas de veto y con total disponibilidad de información sobre las intenciones de todos los Gobiernos (Moravcsik, 1993: 498). Por el contrario, durante la crisis las negociaciones no fueron voluntarias, sino que fueron negociaciones duras basadas en la "asimetría de poder» (Sacchi, 2015), en las amenazas de veto y de salida del euro (Schimmelfennig, 2015a, 2015b) y en la existencia de "condicionalidades disciplinarias» (Ryner, 2015: 282). Se argumenta aquí que la forma específica en la que se desarrolló la condicionalidad tiene que ver con un cambio ideológico basado en el refuerzo del neoliberalismo. Como explica Ryner, el hecho de que algunos Gobiernos consideraran como causa y no como síntoma de la crisis el aumento del déficit público, pretende «extender la estrategia de acumulación basada en una variante particular del neoliberalismo, el ordoliberalismo" (Ryner, 2015: 281). Además, el giro hacia el neoliberalismo se reflejó también en el contenido de las reformas demandadas por las instituciones europeas ya que las políticas sociales y del mercado laboral se consideraron las principales variables de ajuste (Petmesidou y Guillén, 2015). 
El intergubernamentalismo neoliberal y los cambios en la estructura de oportunidad politica

FIgURA 3.

NUEVO RÉGIMEN POLÍTICO

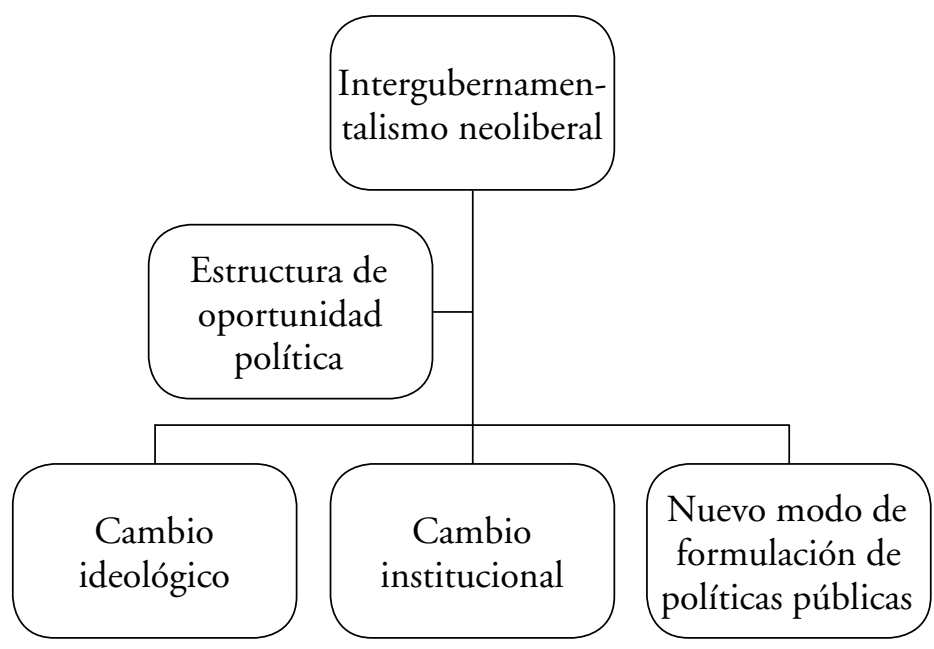

Fuente: elaboración propia.

El INL impactó en la estructura de oportunidad política tanto por el cambio institucional como por el cambio ideológico. Primero, mientras que antes de la crisis las políticas sociales y del mercado laboral eran responsabilidad de los Estados miembros, durante la crisis estas decisiones se tomaron en espacios intergubernamentales. Según la literatura sobre la europeización, que analiza el impacto de la UE en las políticas nacionales, antes de la crisis la influencia de la UE para fijar objetivos comunes en torno a políticas sociales y laborales era moderada, ya que se basaba en la aplicación voluntaria de recomendaciones en torno al Método Abierto de Coordinación. Por el contrario, durante la crisis la UE aumentó la vigilancia sobre los Estados miembros en torno a estas reformas (De la Porte y Heins, 2014). Las decisiones no se tomaron, pues, ni en espacios supranacionales ni nacionales, sino intergubernamentales.

Segundo, el refuerzo del neoliberalismo durante la crisis contradice la principal premisa de la teoría liberal de formación de preferencias nacionales en la cual se basa la teoría de Moravcsik. Esta teoría argumenta que la formación de los intereses nacionales es el resultado del conflicto político doméstico, el cual se produce cuando los diferentes grupos de una sociedad compiten por influencia política. En las democracias liberales, los grupos de interés articulan sus preferencias y después los Gobiernos las agregan a través de mecanismos institucionales y las prácticas de la representación política (Moravcsik, 1993). En este sentido, el INL es compatible con sistemas de 
agregación de intereses de estilo corporativista, ya que reconoce que las preferencias de los grupos de interés imponen «limites legítimos en los mercados» (Moravcsik, 1997: 527).

Por el contrario, en un escenario neoliberal los sindicatos son considerados como obstáculos para conseguir objetivos económicos relacionados con la inflación (Streeck, 2011). Para los economistas neoliberales, la competición política propia de las democracias representativas, de la que forman parte los procesos corporativistas, son los responsables del déficit público (Blyth, 2013). Esto hace que un escenario neoliberal sea incompatible con una estructura de oportunidad corporativista (o de concertación) en las que los derechos laborales y/o políticos de los sindicatos están garantizados. Por el contrario, los principios económicos neoliberales contradicen los derechos de participación de los sindicatos (Scharpf, 1991). De esta manera, un contexto neoliberal está relacionado con una EOP pluralista en la que los sindicatos funcionan como grupos de interés. En este caso, sus demandas no están orientadas a las acción política o laboral en el ámbito de la empresa (como ocurre en sistemas corporativistas) sino a las demandas de derechos civiles, más parecidas a los repertorios de acción utilizados por los movimientos sociales (Gentile, 2015).

\section{UN NUEVO MODO DE FORMULACIÓN DE POLÍTICAS PÚBLICAS: EL JUEGO A DOS NIVELES}

Por último, se argumenta aquí que el INL dio lugar a la aparición de un nuevo modo de formulación de políticas públicas basado en un juego a dos niveles. La teoría del juego a dos niveles fue propuesta por Putnam para explicar la resolución de conflictos internacionales entre Estados (Putnam, 1988). Más tarde, Moravcsik argumentó que el juego a dos niveles ofrece más poder a los Gobiernos respecto a los grupos de intereses domésticos (Moravcsik, 1993, 1997). La condicionalidad habría tenido un efecto paradójico. Si bien por una parte limitó el margen de maniobra de los países del Sur de Europa a través de la imposición de una sola salida de la crisis -la devaluación interna (Armingeon y Baccaro, 2012)-, por otra parte, empoderó a los ejecutivos que querían implementar dichas reformas facilitándoles la expulsión de los sindicatos de los procesos de formulación de políticas públicas (Moury y Freire, 2014).

Durante la crisis de la eurozona, el juego a dos niveles se tradujo en la necesidad de los Gobiernos de responder ante dos tipos de interlocutores con intereses completamente opuestos. Por una parte, estaban los interlocutores externos formados por el grupo de países acreedores del Consejo Europeo liderado por Alemania, junto con el BCE y los mercados financieros. Por otra parte, los Gobiernos tenían que responder a sus interlocutores nacionales, los sindicatos. Este juego a dos niveles modificó los incentivos y los recursos de los que disponen los Gobiernos para iniciar procesos de concertación con los sindicatos (González Begega y Luque Balbona, 2015). Respecto a los incentivos, los Gobiernos tenían que decidir si los beneficios de acordar las políticas de ajuste con los sindicatos eran mayores a los beneficios de implementarlas de 
manera unilateral. En este sentido, las decisiones de los Gobiernos ya no se basaban solo en cálculos electorales, sino también en la necesidad de ganar credibilidad ante los interlocutores externos, lo cual se convirtió en una fuente de legitimidad alternativa (González Begega y Luque Balbona, 2015: 2). En este sentido, los Gobiernos no han tenido que recurrir a los sindicatos para "evitar la culpa», sino que han utilizado estrategias basadas en el «no hay alternativa» (León, Pavolini y Guillén, 2015). Respecto a los recursos, el contenido neoliberal de las reformas basadas en recortes de las políticas sociales y la desregularización de derechos laborales, limitó en gran medida la posibilidad de ofrecer concesiones a los sindicatos a cambio de su consenso.

\section{"En misa y repicando»: cómo el Gobierno de Rodríguez Zapatero respondió a los dos interlocutores}

La condicionalidad europea se desarrolló durante el Gobierno del PSOE de manera informal. Los interlocutores externos pidieron al expresidente socialista José Luis Rodríguez Zapatero en varios ocasiones la aplicación de medidas de austeridad a cambio de no tener que pedir el rescate. Sin embargo, los incentivos para incluir a los sindicatos no desaparecieron del todo durante el Gobierno socialista debido a cuestiones internas del partido. Podemos destacar tres episodios en los procesos de formulación de políticas públicas entre 2010 y 2011. Primero, la implementación de la reforma laboral de junio de 2010 de forma unilateral. En mayo de 2010 se produce la reunión del Consejo Europeo que aprobó el primer rescate de Grecia. Los países acreedores cedieron a cambio de que los deudores aprobaran drásticas medidas de austeridad, las cuales fueron discutidas durante el Ecofin celebrado el 9 de mayo.

La subida del desempleo en España era tema de conversación entre los líderes europeos, por lo que la reforma laboral se convirtió en una de las más discutidas. De hecho, Zapatero ya había hablado con Merkel de la reforma laboral el 6 de mayo antes de la reunión del Consejo (Rodríguez Zapatero, 2013). Ya en España, el expresidente socialista informó a los sindicatos el 13 de mayo de la implementación del paquete de reformas estructurales para reducir el déficit entre las que se encontraba el recorte del sueldo de los funcionarios, aunque mostró su voluntad de que la reforma laboral fuese consensuada con los agentes sociales. Sindicatos y patronal venían negociando la reforma laboral desde 2009 y, aunque habían realizado una declaración de principios conjunta, las posibilidades de un pacto se habían visto mermadas por las posiciones maximalistas de la CEOE y la falta de un equipo de gobierno dentro del Ministerio de Trabajo que facilitara las negociaciones ${ }^{2}$.

En enero de 2010, el Gobierno inició una nueva ronda de negociaciones, pero las posturas encontradas de empresarios y sindicatos frustraron el acuerdo. Tras la ruptura de las negociaciones, y una vez que los sindicatos no eran un obstáculo para imple-

2. Exjefe de gabinete del Ministerio, entrevista personal, 2016. 
mentar los cambios que pedían desde Bruselas, el ala más liberal del PSOE intentó implementar una reforma radical de corte neoliberal, pero el exministro de Trabajo, Celestino Corbacho, intervino para conseguir un resultado más moderado 3 (exasesor parlamentario del PSOE, entrevista personal, 2016). Al final, la reforma se alejaba bastante de lo que pedían desde Europa. Por su parte, CC. OO. y UGT tuvieron que dar una respuesta ante la aprobación unilateral de una reforma de corte desregularizador, pero el carácter moderado de la misma hizo que esa respuesta fuese también comedida. Además, los sindicatos no querían abrir una confrontación continuada con el PSOE porque sabían que había posibilidades de negociar la ya anunciada reforma de las pensiones. De esta manera, los sindicatos convocaron una huelga general para el 29 de septiembre de 2010, una vez que la reforma ya había sido aprobada, contra un ministro ${ }^{4}$ que había anunciado que se iba y haciéndola coincidir con el Día de Acción Europea organizado por la Confederación Europea de Sindicatos (CES).

Desde mayo de 2010, CC. OO. y UGT comprendieron que la formulación de políticas públicas no era ya solo una cuestión de ámbito nacional, sino que había que dar una respuesta a nivel europeo. Por este motivo, la huelga se adaptó a la fecha programada por la CES y se planteó como una protesta contra la idea de austeridad a nivel europeo. La huelga general de 2010 quedó desdibujada hasta tal punto que fue interpretada desde un sector del PSOE no como una huelga contra el Gobierno, sino como una protesta contra la campaña mediática y del Gobierno del PP de Madrid contra los sindicatos ${ }^{5}$. Esto facilitó la apertura de negociaciones de nuevo entre el Gobierno y los sindicatos después de la misma. Por otra parte, al mismo tiempo que la huelga perdía significado como método de presión para echar atrás la reforma, CC. OO. y UGT lanzaron una ILP que pretendía establecer un nuevo marco de relaciones laborales y deshabilitar la reforma laboral de junio de 2010. Aunque la ILP recogió las firmas necesarias y fue admitida a trámite, fue finalmente rechazada por el Congreso en diciembre de 2012.

El segundo episodio se trata del Acuerdo Social y Económico para el Crecimiento, el Empleo y la Garantía de las Pensiones, firmado por los sindicatos, las organizaciones empresariales y el Gobierno en febrero de 2011. Por una parte, desde junio de 2010 hasta el segundo rescate de Grecia de abril de 2011, la prima de riesgo no aumentó drásticamente, relajando así la presión sobre el Gobierno. La condicionalidad había

3. Exasesor parlamentario del PSOE, entrevista personal, 2016.

4. El ministro de Trabajo, Celestino Corbacho, dimitió en agosto de 2011 para ir a formar parte de las listas electorales en Cataluña en las elecciones municipales. Zapatero aprovechó entonces para designar un nuevo ministro, Valeriano Gómez, proveniente del mundo sindical.

5. Exministro de Trabajo del PSOE, entrevista personal, 2016. El mismo Valeriano Gómez, quien sustituyó a Corbacho como ministro de Trabajo, asistió a la manifestación convocada por los sindicatos. 
sido reforzada a través de la creación del Semestre Europeo $^{6}$, pero la prima de riesgo se mantuvo estable durante unos meses. Este relajamiento de la especulación propició que las negociaciones se pudieran desarrollar respetando sus ritmos, dentro del marco del Pacto de Toledo, y sin miedo a la inmediatez de un rescate. Rodríguez Zapatero eligió realizar la reforma de pensiones porque sabía que era eficaz para ganar la credibilidad de la Comisión Europea, de los mercados y de los líderes europeos del grupo de los inversores, en especial la de la canciller alemana, Angela Merkel (Rodríguez Zapatero, 2013). Sin embargo, era difícil convencer a los sindicatos de la necesidad de la misma ya que en aquel momento los fondos de pensiones tenían superávit. Para esto se establecieron mesas de negociación sobre diferentes temas: pensiones, negociación colectiva, políticas activas y energía.

El Acuerdo Social y Económico de 2011 se convirtió en el único pacto quid pro quo de toda la crisis de deuda española. En el intercambio los sindicatos aceptaron el punto más polémico, el incremento de la edad de jubilación a los 67 años. Y a cambio el Gobierno se comprometió a acometer una reforma de la negociación colectiva que no cuestionara el poder sindical ${ }^{7}$. Además, los sindicatos consiguieron una rebaja en el número de años de contribución necesarios para jubilarse de 40 a 38,5, y condiciones favorables para colectivos como los becarios, entre otras. Aun así, ni el Gobierno ni los sindicatos pudieron anunciar el acuerdo como un éxito. La reforma no incluía el desarrollo del factor de sostenibilidad de las pensiones, que era una de las medidas más demandas por los interlocutores externos. Por su parte, los sindicatos no pudieron vender este pacto a sus afiliados como una victoria por la aceptación de los 67 años, lo cual aumentó las críticas internas en los mismos. El juego a dos niveles se hizo patente un día después de la aprobación de la reforma, el 3 de febrero de 2011, cuando en una cumbre bilateral entre Rodríguez Zapatero y Merkel fueron los mismos sindicatos los que explicaron a la canciller las características de la reforma (Rodríguez Zapatero, 2013).

Estos dos episodios impactaron en la capacidad estratégica de los sindicatos para intervenir en la formulación de políticas públicas a nivel nacional. Desde la huelga de 29 de septiembre de 2010, CC. OO. y UGT priorizaron el nivel de acción transnacional, planificando su calendario de protestas en función de los consensos logrados con los sindicatos de otros países dentro de la CES para dar una respuesta a nivel europeo. La apuesta de los sindicatos españoles por el nivel de acción transnacional se vio reforzada en mayo de 2011 cuando en el Congreso de la CES en Atenas, el líder de CC. OO., Ignacio Fernández Toxo, fue elegido como presidente. Esto sirvió a los sindicatos españoles para tener acceso a información y personas desde una posición privilegiada dentro de la $\mathrm{CES}^{8}$.

6. El Semestre Europeo es un ciclo anual a través del cual la UE supervisa los planes presupuestarios y de reformas estructurales de los países.

7. Exministro de Trabajo del PSOE, entrevista personal, 2016

8. Secretario General de CC. OO., entrevista personal, 2015. 
El último episodio empezó con el segundo rescate de Grecia y el rescate de Portugal, tras los cuales España se convirtió de nuevo junto a Italia en el objetivo de la especulación financiera. En la primavera de 2011 la prima de riesgo volvía a subir y era momento de implementar la reforma de negociación colectiva que había servido de contrapartida en el acuerdo de pensiones. Ya en la reunión con Merkel en febrero, la líder alemana había insistido en desligar el aumento salarial de la inflación y ligarlo a la productividad de la empresa (El País, 2011). De nuevo la reforma, que fue aprobada por decreto ley en junio de 2011, no logró conseguir el consenso de empresarios y sindicatos por motivos diversos ${ }^{9}$, ni sirvió para relajar la presión externa sobre España. Ante el aumento drástico de la prima de riesgo, se produjo una nueva intervención de los interlocutores externos. El 5 de agosto de 2011, el expresidente del BCE, Trichet, y Rodríguez Zapatero mantuvieron una conversación telefónica.

Como resultado de la misma se entendía que el BCE empezaría a comprar deuda española a cambio de la implementación de reformas más drásticas. Trichet envió una carta a Zapatero el 5 de agosto de 2011 instándolo a implementar medidas para desregularizar el mercado laboral como la creación de un contrato excepcional que disminuyera considerablemente los costes del despido (Rodríguez Zapatero, 2013). El Gobierno, por su parte, aprobó el Real Decreto del 10 de agosto de 2011 que implementaba nuevas regulaciones respecto al contrato de aprendizaje, el cual pretendía responder a las exigencias del expresidente del BCE. Sin embargo, el decreto no tuvo repercusiones profundas y se consideró de corto impacto. Otro aspecto interesante de este episodio es que el intercambio entre Trichet y Rodríguez Zapatero dio lugar a una nueva interacción entre los dos interlocutores a los que tenía que responder el Gobierno, el BCE y los sindicatos. El expresidente socialista pidió a CC. OO. y UGT la realización de una declaración que tranquilizase al expresidente de BCE y estos escribieron un breve comunicado en los que se comprometían a continuar con las políticas de moderación salarial (Rodríguez Zapatero, 2013).

Respecto al conflicto social contra las medidas de austeridad, en la primavera de 2011 este estaba canalizado por el movimiento de los indignados y el 15M. La capacidad de estos movimientos sociales para conectar con amplias capas de la población difíciles de organizar para los sindicatos, como el «precariado» y los desempleados, dio lugar a varios episodios de protesta masiva. Aunque en principios recelosos del papel institucional de los sindicatos, al final activistas de los movimientos sociales y sindicalistas acabaron fusionándose en espacios comunes como las mareas en defensa de los servicios públicos (Pastor, 2013).

9. Los empresarios no quisieron firmar porque tras los resultados electorales de las elecciones municipales pensaban que el PP ganaría las elecciones generales y que legislaría a su favor (Exministro de Trabajo, entrevista personal, 2016). Por su parte, la reforma no fue pactada con los sindicatos por la existencia de un punto controvertido respecto a la preponderancia de los convenios de empresas sobre los regionales, aunque fue apoyada mayoritariamente por los mismos (Exjefe de gabinete del Ministerio de Trabajo, entrevista personal, 2016). 


\section{El unilateralismo del PP o cómo eliminar a uno de los interlocutores}

Los mecanismos por los que se desarrolló la condicionalidad durante el Gobierno del Partido Popular, que ganó las elecciones generales en noviembre de 2011 con mayoría absoluta, difieren ligeramente de los desarrollados durante el Gobierno de Zapatero. Si bien la condicionalidad siguió dándose en espacios informales, adoptó un carácter cuasiformal en tiempos de Rajoy. Esto se debe al rescate bancario de junio de 2012, que supuso un aumento de la vigilancia por parte de los interlocutores externos de las políticas del mercado laboral y de pensiones que se implementaban en España. Las recomendaciones sobre reformas estructurales del Consejo Europeo empezaron a adoptar un estilo más directo señalando, por ejemplo, que el gasto público relacionado con las pensiones se tenía que recortar más (Recomendaciones del Consejo Europeo, $2011)^{10}$. Además, los decretos leyes aprobados por el PP entre 2012 y 2013 para modificar el sistema de pensiones justificaban la implementación de las reformas en función de las demandas externas.

El reforzamiento de la condicionalidad, la mayoría absoluta y el consenso dentro del PP sobre la idoneidad las medidas de austeridad dieron lugar a la desaparición de incentivos para incluir a los sindicatos. Además, las reformas del mercado laboral y de las pensiones aprobadas por el Gobierno de centro-derecha se basaron en la eliminación de los recursos previamente concedidos a los sindicatos por el Gobierno socialista. Así, la reforma laboral del PP de marzo de 2012 atacaba directamente al poder sindical, mientras que uno de los mayores cambios en las pensiones fue la creación del factor de sostenibilidad que había sido evitado por el PSOE en el acuerdo de 2011. El PP optó por una estrategia de exclusión de los sindicatos de todos los procesos de formulación de política pública, también en lo referente a las reformas de mercado laboral. Después de ganar las elecciones y antes de formar Gobierno, Rajoy mantuvo una reunión con sindicatos y empresarios en las que les comunicó su intención de implementar una reforma laboral. El presidente instó a los agentes sociales a llegar a un acuerdo y así lo hicieron. Sindicatos y empresarios firmaron un acuerdo para la negociación colectiva en enero de 2012 que incluía varias medidas para facilitar la flexibilidad interna de las empresas. Sin embargo, solo unas semanas más tarde el PP implementó de manera unilateral el Real Decreto Ley 3/2012, de 10 de febrero, de Medidas Urgentes para la Reforma del Mercado Laboral. CC. OO. y UGT convocaron una huelga general para el 29 de marzo de 2012. Por aquel entonces los sindicatos españoles ya habían tomado la iniciativa dentro de la CES para poder coordinar un día de huelga europeo con otros sindicatos de países del Sur de Europa. Para esa ocasión los sindicatos del Sur de Europa no pudieron consensuar una fecha. La CES acabó convocando un día de acciones descentralizadas el 29 de febrero de 2012 durante el

10. «Recommendation for a Council Recommendation on the National Reform programme 2011 of Spain» Disponible aquí: http://ec.europa.eu/europe2020/pdf/recommendations_2011/csr_ spain_en.pdf 
cual los sindicatos españoles realizaron 24 manifestaciones en diferentes ciudades españoles, además de una concentración en frente de la representación de la Comisión Europea en Madrid.

Los sindicatos aumentaron también la presión institucional transnacional y mantuvieron una reunión con la canciller alemana, Angela Merkel, en julio de 2012, cuyo objetivo era desmentir los rumores que habían llegado a la canciller sobre el papel negativo de los sindicatos españoles para la implementación de las reformas estructurales $^{11}$. Durante el encuentro, que fue facilitado por los sindicatos alemanes, CC. OO. y UGT mostraron a Merkel el acuerdo al que habían llegado con los empresarios para convencerla de que no había motivos que justificasen el rechazo de Rajoy al diálogo social. Hacía un año que el presidente del PP no recibía a los sindicatos y estos querían convencer a Merkel de que Rajoy estaba equivocado al adoptar una política que excluía a los agentes sociales. Una semana después de la reunión con la canciller, Rajoy recibió a los sindicatos para informarles de las medidas que se iban a tomar, pero no se iniciaron negociaciones.

La presencia de la secretaria de la CES, Bernadette Ségol, también se incrementó a partir de 2012. En agosto de 2012 Ségol intervino en la rueda de prensa en la que los sindicatos españoles anunciaron la puesta en marcha de un proceso movilizador basado en dos ideas. Una, la realización de manifestaciones que incluyeran a capas más amplias de la sociedad a través de la creación de la Cumbre Social. Y dos, el inicio de una campaña de movilización para la realización de un referéndum contra las medidas de austeridad. Al mismo tiempo que los sindicatos desarrollaban estas estrategias encaminadas a politizar a sectores más amplios de la sociedad civil, seguían ejerciendo de lobby dentro de la CES para conseguir un día de acción conjuntos. La CGTP portuguesa había decidido convocar una huelga general para el 14 de noviembre de 2012 y, junto con CC. OO. y UGT, presionaron dentro de la CES para que ese se convirtiera en una fecha de consenso. Otros sindicatos del Sur de Europa, como la CGIL italiana y los sindicatos griegos, acabaron sumándose a la convocatoria de huelga. Finalmente, la CES asumió el 14 de noviembre de 2012 como día de acción conjunto, pero la participación del resto de sindicatos fue dispar. En España una de las claves de la huelga fue también su carácter de protesta social y la participación del $15 \mathrm{M}$ en la misma (Pastor, 2013).

Aun así, el Gobierno de Rajoy siguió con las políticas unilaterales y aprobó el Real Decreto Ley 28/2012, de 30 de noviembre de 2012, que suponía la congelación de las pensiones para el 2013 y la eliminación de una de las pagas extra. El 22 de marzo de 2013, el PP aprobó también el Real Decreto Ley 5/2013, que modificaba las condiciones para acceder a la jubilación anticipada limitando el acceso a la misma. Las referencias a la condicionalidad europea eran especialmente explícitas en este último, que

11. Secretario General de CC. OO., entrevista personal, 2015. 
apuntaba a las estrategias de Europa 2020 y al libro blanco de la Comisión Europea ${ }^{12}$ como referentes en el diseño de la misma.

La última intervención del Gobierno del PP en el sistema de pensiones empezó en abril 2013 cuando formó un comité de expertos para la creación del factor de sostenibilidad. Aunque los sindicatos intentaron que se convocara el Pacto de Toledo para negociar la reforma, esta fue finalmente aprobada también de forma unilateral. Por su parte los sindicatos realizaron un viraje estratégico en 2013 en el que decidieron bajar el nivel de conflictividad y no convocar más huelgas generales, las cuales no estaban dando sus frutos, con el objetivo de reestablecer el diálogo social con el Gobierno. Esto supuso también bajar la presión dentro de la CES para realizar acciones colectivas conjuntas $^{13}$. Aun así, CC. OO. y UGT no olvidaron el plano transnacional. Dentro de las acciones para reabrir el diálogo con el Gobierno, los sindicatos optaron también por acciones transnacionales basadas en la presión institucional. En esta nueva reorientación se enmarcó la reunión que en junio de 2013 los sindicatos españoles y la exsecretaria general de la CES, Bernadette Ségol, tuvieron con el presidente Rajoy antes de la cumbre de los jefes de Estado para presentarle el plan de inversiones y el plan para el empleo juvenil diseñados por la CES. Sin embargo, el diálogo social no se retomó hasta el 2014, pocos meses después que España saliese del rescate bancario en noviembre de 2013.

\section{CONCLUSIONES}

Este artículo ha tenido como objetivo explicar por qué los sindicatos españoles desarrollaron estrategias transnacionales y de carácter civil, junto a las respuestas tradicionales de diálogo social y huelgas generales. Para responder a esta cuestión, el artículo ha propuesto un nuevo marco analítico basado en el concepto de estructura de oportunidad política. Se ha argumentado aquí que la condicionalidad de la UE dio lugar a la aparición de un nuevo régimen de adopción de decisiones que hemos denominado «intergubernamentalismo neoliberal» por el tipo de cambios institucionales e ideológicos que trajo consigo. Este régimen político impactó en la estructura de oportunidad política por dos motivos. Primero, el giro hacia el intergubernamentalismo dio lugar a que las decisiones respecto a las políticas sociales y laborales no se tomaran en los espacios de decisión supranacionales y nacionales habituales. Segundo, el refuerzo de la ideología neoliberal modificó la forma en la que los Gobiernos negocian la coordinación de las políticas a nivel europeo.

Además, el giro hacia el neoliberalismo cuestionó el papel de las democracias representativas y de los procesos corporativistas para decidir las políticas para salir de

12. Comisión Europea (2012). Libro blanco «Agenda para unas pensiones adecuadas, seguras y sostenibles", COM (2012) 55 final.

13. Secretario General de CC. OO., entrevista personal, 2015. 
la crisis. Todos estos cambios dieron lugar a un nuevo modo de formulación de políticas públicas basado en un juego a dos niveles. Los Gobiernos tuvieron que atender a las demandas de los interlocutores externos y de los sindicatos. La reducción de incentivos y de recursos disponibles para la inclusión de los sindicatos obstaculizó la puesta en marcha de procesos corporativistas. Además, la consecuencia no fue solo un aumento del unilateralismo, sino la aparición de un nuevo tipo de estrategia de los Gobiernos que podríamos llamar «consulta instrumental». En varias ocasiones, los sindicatos se convirtieron en actores clave para que los Gobiernos consiguieran credibilidad ante los interlocutores externos, como la presentación del acuerdo de pensiones a Angela Merkel o la declaración de moderación salarial para convencer al expresidente del BCE, Jean-Claude Trichet.

Todos estos cambios tuvieron un impacto en las decisiones estratégicas de los sindicatos. La acción política para influir a los Gobiernos nacionales cambió de significado. Las huelgas generales adoptaron un carácter transnacional para responder a la intervención de la UE en políticas nacionales, y en su desarrollo fue fundamental la politización de amplios sectores de la sociedad civil. El nuevo régimen político dio lugar también a una estructura de oportunidad política pluralista, lo cual hizo que los sindicatos actuaran más como grupo de presión utilizando repertorios propios de los movimientos sociales. Por último, aunque el caso español nos ha servido para ilustrar un mecanismo general, sería adecuado que futuras investigaciones observen si se han desarrollado los mismos procesos en otros países. Además, futuros estudios tendrán que analizar si el nuevo régimen político fue un resultado coyuntural de la crisis o si sus dinámicas políticas han continuado después de la misma.

\section{Bibliografia}

Afonso, Alexander. 2013. Social Concertation in Times of Austerity. European Integration and the Politics of Labour Market Reforms in Austria and Switzerland. Amsterdam: Amsterdam University Press.

Armingeon, Klaus y Lucio Baccaro. 2012. «Political economy of the sovereign debt crisis: the limits of internal devaluation», Industrial Law Journal, 41 (3): 254-275. Disponible en: http://dx.doi.org/10.1093/indlaw/dws029

Avdagic, Sabina, Martin Rhodes y Jelle Visser (eds.). 2011. Social Pacts in Europe: Emergence, Evolution and Institutionalization. Oxford: Oxford University Press.

Baccaro, Lucio. 2003. "What is Alive and What is Dead in the Theory of Corporatism", British Journal of Industrial Relations, 41 (4): 683-706. Disponible en: http://dx.doi.org/10.1046/j.1467-8543.2003.00294.x

Barranco, Oriol y Oscar Molina. 2014. «Sindicalismo y crisis económica: amenazas, retos y oportunidades», Kultur, 1 (2): 171-194. Disponible en: http://dx.doi. org/10.6035/kult-ur.2014.1.2.9. 
Beach, Derek y Rasmus Brun Pedersen. 2013. Process-Tracing methods. Foundations and guidelines. Ann Arbor: The University of Michigan Press. Disponible en: http://dx.doi.org/10.3998/mpub.2556282.

Bieler, Andreas y Adam David Morton (eds.). 2001. Social Forces in the Making of the New Europe. The restructuring of European Social Relations in the Global Political Economy. UK: Palgrave Macmillan.

Blyth, Mark. 2013. Austerity. The History of a dangerous idea. Oxford: Oxford University Press.

Campos Lima, María da Paz y Antonio Martín Artiles. 2011. «Crisis and trade unions challenges in Portugal and Spain: between general strikes and social pacts", Transfer: European Review of Labour and Research, 17 (3): 387-402. Disponible en: http://dx.doi.org/10.1177/1024258911410806.

Campos Lima, Maria da Paz y António Martín Artiles. 2014. «Descontentamento na Europa em tempos de austeridade: Da ação coletiva à participação individual no protesto social», Revista Critica de Ciências Sociais, 103: 137-172. Disponible en: http://dx.doi.org/10.4000/rccs.5569.

Cerrillo Vidal, José Antonio. 2013. «From general strike to social strike: movement alliances and innovative actions in the November 2012 Spanish general strike», Interface: A Journal for and About social movements, 5 (2): 39-46.

De la Porte, Caroline y David Natali. 2014. "Altered Europeanisation of Pension Reform in the Context of the Great Recession: Denmark and Italy Compared", West European Politics, 37 (4): 732-749. Disponible en: http://dx.doi.org/10.108 0/01402382.2014.919770.

De la Porte, Caroline y Elke Heins. 2014. "Game change in EU social policy: Towards more European ntegration", en M. J. Rodrigues and E. Xiarchogiannopoulou (eds.), The Eurozone Crisis and the Transformation of EU Governance. Aldershot, UK: Ashgate.

Degryse, Christophe. 2012. The new European economic governance. ETUI Working Paper, 2012/14.

Dribbusch, Heiner. 2015. "Where is the European general strike? Understanding the challenges of trans-European trade union action against austerity», Transfer: European Review of Labour and Research, 21 (2): 171-185. Disponible en: http://dx. doi.org/10.1177/1024258915573185.

Ebbinghaus, Bernhard. 2011. "The role of trade unions in European pension reforms: From 'old' to 'new' politics?», European Journal of Industrial Relations, 17 (4): 315331. Disponible en: http://dx.doi.org/10.1177/0959680111420208.

El País. (2011). «Merkel en Madrid», El País, editorial, 4-2-2011. Disponible en: Disponible en: http://elpais.com/diario/2011/02/04/opinion/1296774001_850215.html.

Erne, Roland. 2008. European unions: labor's quest for a transnational democracy. Nueva York: Cornell University Press.

Gentile, Antonina. 2015. "Labor repertoires, neoliberal regimes and US hegemony: what 'deviant'Italy tells us of OECD unions'paths to power», European Political 
Science Review, 7: 243-270. Disponible en: http://dx.doi.org/10.1017/ S1755773914000101.

González Begega, Sergio y David Luque Balbona. 2014. «Goodbye to competitive corporatism in Spain? Social Pacting and conflict in the economic crisis», Revista Española de Investigaciones Sociológicas, 148: 79-102.

González Begega, Sergio y David Luque Balbona. 2015. «Crisis económica y deterioro de los pactos sociales en el Sur de Europa», Revista Internacional de Sociología, 73 (2):1-13.

Haas, E. B. 1958. The uniting of Europe: Political, social, and economic forces, 19501957. Stanford: Stanford University Press.

Hall, Peter A. y David Soskice (eds.). 2001. Varieties of capitalism: The institutional foundations of comparative advantage. Oxford: Oxford University Press.

Hamann, Kerstin. 2012. The Politics of Industrial relations. Labor unions in Spain. New York: Routledge.

Hamann, Kerstin y John Kelly. 2007. «Europe Party Politics and the Reemergence of Social Pacts in Western", Comparative Political Studies, 40: 971. Disponible en: http://dx.doi.org/10.1177/0010414006294818.

Helle, Idar. 2015. «A new proletariat in the making? Reflections on the 14 November 2012 strike and the movements of 1968 and 1995», Transfer: European Review of Labour and Research, 21(2): 229-242. Disponible en: http://dx.doi. org/10.1177/1024258915573388.

Hyman, Richard. 2001. Understanding European trade unionism: between market, class and society. London: SAGE Publications.

Hyman, Richard. 2005. "Trade unions and the politics of the European Social Model», Economic and Industrial Democracy, 26 (1):9-40. Disponible en: http://dx. doi.org/10.1177/0143831X05049401.

Hyman, Richard. 2010. Social dialogue and industrial relations during the economic crisis: Innovative practices or business as usual? International Labor Office, Dialogue Working Paper, 11.

Hyman, Richard y Rebecca Gumbrell-McCormick. 2010. «Trade unions, politics and parties: is a new configuration possible?», Transfer: European Review of Labour and Research, 16 (3): 315-331. Disponible en: http://dx.doi. org/10.1177/1024258910373863.

Kelly, John. y Kerstin Hamann. 2004. «Unions as political actors: a recipe for revitalization?», en Carola M. Frege and John Kelly (eds.), Varieties of Unionism: Strategies of Union Revitalization in a Globalizing Economy. Oxford: Oxford University Press.

Kitschelt, Herbert P. 1986. «Political opportunity structures and political protest: anti-Nuclear Movements in four democracies», British Journal of Political Science, 16(1): 57-85. Disponible en: http://dx.doi.org/10.1017/S000712340000380X.

León, Margarita, Emmanuela Pavolini y Ana M. Guillén. (2015). «Welfare rescaling in Italy and Spain: political strategies to deal with harsh austerity", European Journal of Social Security, 17 (2): 182- 201. 
Luque Balbona, David y Sergio González Begega. 2015. «Austerity and welfare reform in south-western Europe. A farewell to corporatism in Italy, Spain and Portugal?», European Journal of Social Security, 17 (2): 272-292.

Molina, Óscar. 2005. «Political exchange and bargaining reform in Italy and Spain», European Journal of Industrial Relations, 11 (1): 7-26. Disponible en: http://dx. doi.org/10.1177/0959680105050397.

Molina, Óscar y Fausto Miguélez. 2013. From negotiation to imposition: Social dialogue in austerity times in Spain. International Labour Organization, Working Paper, 51.

Molina, Óscar y Martin Rhodes. 2002. "Corporatism: The past, Present, and future of a Concept», Annual Review of Political Science, 5 (1): 305-31. Disponible en: http://dx.doi.org/10.1146/annurev.polisci.5.112701.184858

Moravcsik, Andrew. 1993. «Preferences and Power in the European Community: A Liberal Intergovernmentalist Approach", Journal of Common Market Studies, 31(4): 473-524. Disponible en: http://dx.doi.org/10.1111/j.1468-5965.1993. tb00477.x.

Moravcsik, Andrew. 1997. "Taking preferences seriously: a liberal theory of international politics», International Organization, 51 (4): 513-53. Disponible en: http:// dx.doi.org/10.1162/002081897550447

Moury, Catherine y Andrè Freire. 2013. «Austerity Policies and Politics: the case of Portugal», Pôle Sud, 2 (2013): 35-56.

Natali, David y Philippe Pochet. 2009. «The evolution of Social Pacts in the EMU Era: What Type of Institutionalization?», European Journal of Industrial Relations, 15 (2): 147-166. Disponible en: http://dx.doi.org/10.1177/0959680109103606.

Oliet Palá, Alberto. 2004. La concertación social en la democracia española: crónica de un dificil intercambio. Valencia: Tirant Lo Blanc.

Pastor, Jaime. 2013. «El movimiento 15m en Madrid, 2012», Anuario del Conflicto Social, 1(1): 205-214.

Petmesidou, María y Ana M. Guillén. 2015. Economic crisis and austerity in Southern Europe: threat or opportunity for a sustainable welfare state? OSE Research Paper, 18.

Princen, Sebastiaan y Bart Kerremans. 2008. «Opportunity structures in the EU multilevel system», West European Politics, 31 (6): 1129-1146. Disponible en: http:// dx.doi.org/10.1080/01402380802370484

Putnam, Robert D. 1988. «Diplomacy and domestic politics. The logic of two-level game», International Organization, 42 (3): 427-460. Disponible en: http://dx.doi. org/10.1017/S0020818300027697

Rodríguez Zapatero, José Luis. 2013. El Dilema: 600 días de vértigo. Barcelona: Planeta.

Ryner, Magnus. 2015. «Europe's ordoliberal iron cage: critical political economy, the euro crisis and its management», Journal of European Public Policy, 22 (2): 275294. Disponible en: http://dx.doi.org/10.1080/13501763.2014.995119 
Sacchi, Stefano. 2015. "Conditionality by other means: EU involvement in Italy's structural reforms in the sovereign debt crisis», Comparative European Politics, 13 (1): 77-92. Disponible en: http://dx.doi.org/10.1057/cep.2014.42

Scharpf, Fritz W. 1991. Crisis and choice in European Social Democracy. Ithaca: Cornell University Press.

Scharpf, Fritz W. 2002. The European social model: coping with the challenges of diversity. Max-Planck Institut für Gesellschaftsforschung Working Paper. 2002/8. Köln: MPIfG.

Schimmelfenning, Frank. 2015a. «Liberal intergovernmentalism and the euro are crisis», Journal of European Public Policy, 22 (2): 177-195. Disponible en: http://dx. doi.org/10.1080/13501763.2014.994020.

Schimmelfennig, Frank. 2015b. "What's the news in 'new intergovernmentalism'? A critique of Bickerton, Hodson and Puetter", Journal of Common Market Studies, 53 (4), 723-730. Disponible en: http://dx.doi.org/10.1111/jcms.12234.

Schmitter, Philippe. C. 1974. "Still the century of corporatism?», The Review of Politics, 36 (01): 85-131. Disponible en: http://dx.doi.org/10.1017/ S0034670500022178.

Streeck, Wolfgang. 2011. The Crisis in Context. Democratic Capitalism and its Contradictions. MPIfG Discussion Paper, 11/15.

Streeck, Wolfgang y Anke Hassel. 2003. International handbook of trade unions. Edward Elgar Publishing.

Tilly, Charles. 1995. Popular Contention in Great Britain. 1758-1834. Cambridge, MA: Harvard University Press.

Tilly, Charles.2006. Regimes and repertoires. Chicago: University of Chicago Press. Disponible en: http://dx.doi.org/10.7208/chicago/9780226803531.001.0001.

Tilly, Charles y Sidney Tarrow. 2015. Contentious politics. Oxford: Oxford University Press.

Urban, Hans-Jürgen. 2012. "Crisis corporatism and trade union revitalization in Europe», en $A$ triumph of failed ideas: European models of capitalism in the crisis. Bruselas: ETUI.

Weaver, R. Kent. 1986. "The Politics of Blame Avoidance», Journal of Public Policy, 6 (4): 371-98. Disponible en: http://dx.doi.org/10.1017/S0143814X00004219.

Presentado para evaluación: 5 de julio de 2016.

Aceptado para publicación: 27 de octubre de 2016.

\section{ANGIE GAGO}

angiegagoflores@gmail.com

Es candidata al doctorado de Estudios Políticos en la Universidad de Milán y asistente de investigación en el proyecto «Democracy in Times of Crisis: Power and Discourse in a three-level game» en la Facultad de Ciencias Sociales y Humanidades de la 
Universidad Nueva de Lisboa (FCSH-NOVA). También es miembro de otros proyectos de investigación como REScEU (Reconciling Economic and Social Europe) en la Universidad de Milán. Recientemente ha contribuido en la obra colectiva Desafios del Estado de bienestar en Noruega y España, coordinado por Francisco J. Moreno y Eloísa Del Pino, 2015. 